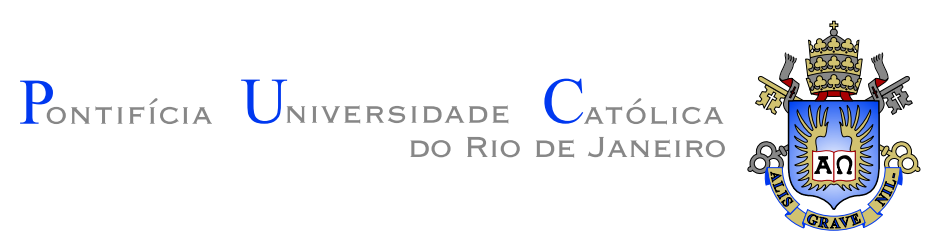

Alexander Argüello Quiroga

\title{
Impacto do fundo cósmico de neutrino na expansão do Universo
}

\section{Dissertação de Mestrado}

Dissertação apresentada como requisito parcial para obtenção do grau de Mestre pelo Programa de Pós-graduação em Física do Departamento de Física da PUC-Rio

Orientador : Prof. Hiroshi Nunokawa

Co-Orientador: Prof. Martin Makler

Rio de Janeiro

junho de 2009 


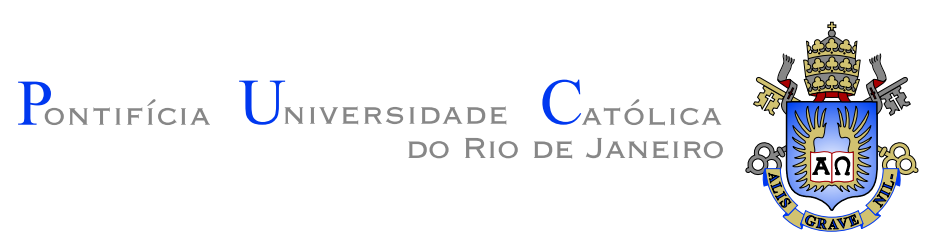

Alexander Argüello Quiroga

\section{Impacto do fundo cósmico de neutrino na expansão do Universo}

Dissertação apresentada como requisito parcial para obtenção do grau de Mestre pelo Programa de Pós-graduação em Física do Departamento de Física do Centro Técnico Científico da PUCRio. Aprovada pela Comissão Examinadora abaixo assinada.

Prof. Martin Makler

Co-Orientador ICRA-CBPF

Prof. Orlando Luis Goulart Peres

UNICAMP

Prof. Carla Göbel Burlamaqui de Mello

Departamento de Física-PUC-Rio

Prof. José Eugenio Leal

Coordenador Setorial do Centro Técnico Científico - PUC-Rio

Rio de Janeiro, 15 de junho de 2009 
Todos os direitos reservados. É proibida a reprodução total ou parcial do trabalho sem autorização da universidade, do autor e do orientador.

\section{Alexander Argüello Quiroga}

Graduou-se em física na Universidad Industrial de Santander em 2006.

Ficha Catalográfica

Quiroga, Alexander Argüello

Impacto do fundo cósmico de neutrino na expansão do Universo / Alexander Argüello Quiroga; orientador: Hiroshi Nunokawa; co-orientador: Martin Makler. - Rio de Janeiro : PUC-Rio, Departamento de Física, 2009.

v., 102 f: il. ; $29,7 \mathrm{~cm}$

1. Dissertação (mestrado) - Pontifícia Universidade Católica do Rio de Janeiro, Departamento de Física.

Inclui referências bibliográficas.

1. Física - Tese. 2. Neutrino. 3. Massa do neutrino. 4. Distância de Luminosidade. 5. Neutrinos Cosmológicos. I. Nunokawa,Hiroshi. II. Makler, Martin. III. Pontifícia Universidade Católica do Rio de Janeiro. Departamento de Física. IV. Título.

CDD: 510 


\section{Agradecimentos}

À Deus, Criador de toda a ciência e conhecimento;

Aos meus orientadores Hiroshi Nunokawa e Martin Makler, professores que admiro não somente por seu alto gráu de conhecimento, também pela capacidade de ensino que eles têm, pela paciência que tiveram na realização deste trabalho, por terem me aceitado como seu estudande e pela preocupação que eles tiveram na minha formação como pesquisador;

Quero dar um agradecimento especial ao professor Martin Makler por toda a ajuda, pelo tempo e pela dedicação;

Ao professor Welles Morgado, pelas dúvidas que resolveu na parte de matemática e de conceitos de física;

À Kelly de Carvalho, pelas correções de português que fez, pelo tempo, e por ter oferecido ajuda quando eu mais precisava;

Aos meus amigos e colegas Fábio Alex Pereira dos Santos e Thiago Mühlbeier, pelas discussões em física, em programação e Latex;

À minha amiga Johanna Pacheco, pela ajuda que me deu quando cheguei no Rio de Janeiro;

Aos meus colegas e amigos Jefferson Ferraz, Fernando Mejia, Dunieskys González, por terem me agüentado nestes dois anos;

À Claudia Friacchiolla por ter me apresentado ao professor Martin Makler;

Aos demais alunos e funcionários da Pós-Graduação do Departamento de Física da PUC-Rio, os quais de imnúmeras formas ajudaram no andamento desta dissertação, em especial à Giza e ao Julinho;

À CAPES e ao CNPq pelo apoio financeiro, que foi de vital importância para a elaboração deste trabalho. 


\section{Resumo}

Quiroga, Alexander Argüello; Nunokawa,Hiroshi; Makler, Martin. Impacto do fundo cósmico de neutrino na expansão do Universo. Rio de Janeiro, 2009. 102p. Dissertação de Mestrado Departamento de Física, Pontifícia Universidade Católica do Rio de Janeiro.

Os neutrinos são produzidos copiosamente no Universo primordial e são hoje as espécies mais abundantes de partículas após dos fótons. Com a descoberta da oscilação de neutrinos sabe-se que eles possuem massa. Os efeitos de neutrinos na formação de estruturas têm sido muito estudados e a comparação com os dados observacionais establece limites sobre a soma de suas massas. Nesta monografia obordamos outro aspecto dos neutrinos em cosmologia que tem sido pouco estudado na literatura: a sua influência na expansão global e suas possíveis implicações observacionais. Um aspecto interessante dos neutrinos do fundo cósmico é que, dentro dos limites actuais em suas massas, ao menos uma espécie deve ter passado de um regime relativístico a não-relativístico desde o desacoplamento matéria-radiação até o presente. Essa mudança de regime poderia acarretar em efeitos observacionais característicos desse processo. Neste trabalho investigamos a equação de estado dos neutrinos em função de sua massa e sua temperatura. A partir desse resultado, obtemos a taxa de expansão e a distância de luminosidade em função do desvio para o vermelho para um Universo contendo neutrinos massivos, além de matéria escura, constante cosmologica e radiação. Embora espera-se que o impacto dos neutrinos nessas quantidades observáveis seja pequeno, o objetivo deste trabalho é verificar se ele pode ser mensurável no contexto da emergente "cosmologia de presição".

\section{Palavras-chave}

Neutrino; Massa do neutrino; Distância de Luminosidade; Neutrinos Cosmológicos; 


\section{Abstract}

Quiroga, Alexander Argüello; Nunokawa,Hiroshi; Makler, Martin. Impact of the Cosmic Neutrino Background in the Expansion of the Universe. Rio de Janeiro, 2009. 102p. MSc Dissertation - Departamento de Física, Pontifícia Universidade Católica do Rio de Janeiro.

Neutrinos are produced copiously in the primordial Universe and today they are the most abundant specie of particles after the photons. With the discovery of neutrinos oscillation it is known that at least two of them have mass, although the absolute values are unknown. The effects of massive neutrinos in the large scale structure formation have been much studied and the comparison with observational data establishes limits over the neutrinos mass sum. In this dissertation we broach other aspect of neutrinos in cosmology which has been few studied in literature: its influence in the global expansion and the possible observational implications. An interesting aspect of cosmic neutrinos background is that inside the present masses limits at least one specie must have passed from a relativistic regime to a non-relativistic one, since the matter-radiation decoupling until now. This change in regime could cause observational effects characteristics of this process. In this research we investigate the neutrinos state equation in function of their mass and temperature. From this result, we obtain the expansion rate and the luminosity distance in function of the Universe red-shift considering the presence of massive neutrinos, besides the dark matter, cosmological constant and radiation. Even though we expect that the neutrinos impact in these observable be small, the objective of this work is to verify if it can be measured in the context of the emergent "precision cosmology".

\section{Keywords}

Neutrino; Neutrino masses; Luminosity distance; Cosmological Neutrinos; 


\section{Sumário}

1 Introdução 13

2 Neutrinos $\quad \mathbf{1 6}$

2.1 História dos neutrinos 16

2.2 Física de neutrinos 23

2.3 Neutrinos de Majorana e Dirac 27

$\begin{array}{lll}2.4 & \text { Massa do neutrino } & 29\end{array}$

3 Cosmologia $\quad 36$

3.1 Introdução 36

3.2 Elementos de Relatividade Geral 37

3.3 A métrica Robertson-Walker 40

3.4 Propagação da luz 42

3.5 Distância de Luminosidade. 44

3.6 Movimento Geodésico 48

3.7 A função de distribuição dos neutrinos 48

4 A densidade de energia e pressão dos neutrinos $\quad \mathbf{5 6}$

4.1 Introdução $\quad 56$

$\begin{array}{lll}4.2 & \text { Densidade e pressão dos neutrinos } & 57\end{array}$

5 Resultados $\quad 75$

5.1 Parâmetros cosmológicos 76

5.2 Distância de luminosidade $\quad 76$

5.3 Parâmetro de Hubble 83

$\begin{array}{lll}6 & \text { Conclusão } & 91\end{array}$ 


\section{Lista de figuras}

2.1 Gráfico da mistura dos auto-estados de sabor do neutrino $\nu_{e}, \nu_{\mu}, \nu_{\tau}$, dos auto-estados de massa $\nu_{1}, \nu_{2}, \nu_{3}$ e dos ângulos de mistura $\theta_{12}, \theta_{13}, \theta_{23}$.

4.1 Gráfico em escala logarítmica da Densidade de energia e da Pressão em função de $R=m_{\nu} / T_{\nu}$. É possível ver a mundança de comportamento da situação relativística para $R \ll 1$ e a nãorelativística para $R \gg 1$. A diferença entre as curvas em $R \gg 1$ é devida ao fator de $1 / 3$ entre $\rho$ e $P$, lembrando que $w=P / \rho=1 / 3$. 57

4.2 Gráfico comparativo entre a densidade de energia numérica e as aproximações analíticas. Para $R \gg 1$ pode-se ver que a linha vermelha é a curva da densidade de energia feita numericamente, e as linhas verde e azul são as aproximações feitas em primeiro e segundo graus chamadas no gráfico $A p$ e $A p_{2}$, ver (4.13).

4.3 Ampliação da figura (4.2) na região próxima a $R=1$. Podemos ver como $A p$ se afasta da curva numérica $\rho_{\nu}(R)$ mais que $A p_{2}$. Vemos também como as aproximações deixam de ser válidas em $R \simeq 3$.

4.4 Gráfico da equação de estado $w$ em função de $R$.

4.5 Gráfico da equação de estado $w$ junto com as aproximações para primeira e segunda ordem

4.6 Ampliação da figura (4.5) na região de $1 \leq R \leq 100$, onde a aproximação $A p_{2}$ que é quem tem as duas ordens da aproximação da equação linha vermelha é a feita numericamente, a verde é a aproximação em primeira ordem e a azul é a aproximação em segunda ordem, para a aproximação de $R \gg 1$.

4.7 Gráfico onde se apresentam as curvas feitas analiticamente para $R \ll 1$ e numericamente.

4.8 Gráfico do desvio relativo da aproximação da curva numérica. Para $R \ll 1$ o desvio relativo entre $\rho_{n}$ e $A p_{2}$ em (4.14) é quase 1 o que nos diz que a aproximação deixa de ser válida. Com a linha tracejada mostramos o valor e a região do desvio relativo que estamos usando para cortar a função da densidade de energia (4.1). Como estamos considerando a aproximação $R \ll 1$, vemos que estamos no limite onde a função aproximada (4.14) ainda é válida e não tem erros de arredondamento.

4.9 Gráfico do desvio relativo que tem a aproximação da curva numérica. Para $R \gg 1$ o desvio relativo entre $\rho_{n}$ e $\rho_{A p_{2}}$ é quase 1 o que nos diz que a aproximação deixa de ser válida. Com a linha tracejada mostramos o valor e a região do desvio relativo que estamos usando para cortar a função da densidade de energia (4.1). 
4.10 Gráfico da densidade de energia e as aproximações para $R \gg 1$ e $R \ll 1$, onde mostramos a função composta da densidade de energia. Aqui a linha verde é a aproximação para $R \gg 1$ e a linha azul é a aproximação para $R \ll 1$.

4.11 Gráfico da densidade de energia e a pressão com $m=1 \mathrm{eV}$, onde se vê o desvio que têm as curvas em comparação com as funções calculadas com massa $m_{\nu}=0 \mathrm{eV}$.

4.12 Gráfico da densidade de energia em função da temperatura e com diferentes massas do neutrino.

4.13 Ampliação da figura (4.12). Podemos observar que cada curva se afasta da curva $m_{\nu}=0 \mathrm{eV}$ segundo sua massa. Por exemplo, a curva da densidade de energia com $m_{\nu}=0.5 \mathrm{eV}$ se afasta em aproximadamente $T_{\gamma}=0.5 \mathrm{eV}$. O mesmo acontece com as demias curvas.

4.14 Gráfico da equação de estado com massa do neutrino $m_{\nu}=$ $0,0.1,0.5,1,1.5,2 \mathrm{eV}$.

4.15 Gráfico da equação de estado (4.14) em escala logarítmica.

5.1 Gráfico da distância de luminosidade em função do desvio para o vermelho, para diferentes massas dos neutrinos.

5.2 Zoom da Figura (5.1). Neste gráfico observamos um pequeno aumento da distância de luminosidade devida ao térmo de massa considerado na densidade de energia dos neutrinos.

5.3 Gráfico da distância de luminosidade em função da massa dos neutrinos para diferentes desvios para o vermelho.

5.4 Gráfico em três dimensões da distância de luminosidade em função da massa dos neutrinos para um desvio para o vermelho.

5.5 Gráfico da diferença fracionária da distância de luminosidade em função do desvio para o vermelho. Para a análise usamos valores especificos da massa dos neutrinos tais como: $0.10 .51 .01 .52 .0 \mathrm{eV}$, e as comparamos com $m_{\nu}=0 \mathrm{eV}$.

5.6 Gráfico da diferença fracionária da distância de luminosidade em função da massa do neutrino, para diferentes valores da do desvio para o vermelho.

5.7 Isocontorno da distância de luminosidade em função da massa dos nutrinos e do desvio para o vermelho.

5.8 Gráfico em três dimensões da diferença fracionária da distância de luminosidade em função da massa dos neutrinos e do desvio para o vermelho.

5.9 Gráfico da diferença fracionária da razão da distância de luminosidade em função da massa dos neutrinos. 
5.10 Gráfico do Parâmetro de Hubble em função do desvio para o vermelho para diferentes valores da massa do neutrino. Neste gráfico observamos uma pequena disminução do parâmetro de Hubble devida ao térmo de massa considerado na densidade de energia dos neutrinos.

5.11 Gráfico do Parâmetro de Hubble em função do desvio para o vermelho para diferentes valores da massa do neutrino. Neste gráfico observamos uma pequena diminuição do parâmetro de Hubble devida ao termo de massa considerado na densidade de energia dos neutrinos.

5.12 Gráfico do parâmetro de Hubble em função da massa dos neutrinos para diferentes desvios para o vermelho.

5.13 Gráfico em três dimensões do parâmetro de Hubble em função da massa dos neutrinos para um desvio para o vermelho.

5.14 Gráfico da diferença fracionária do Parâmentro de Hubble em função do desvio para o vermelho. Para a análise usamos valores especificos da massa dos neutrinos tais como: $0.10 .51 .01 .52 .0 \mathrm{eV}$, e as comparamos com $m_{\nu}=0 \mathrm{eV}$.

5.15 Gráfico da diferença fracionária do Parâmentro de Hubble em função da massa dos nuetrinos. Para a análise usamos valores especificos do desvio para o vermelho tais como: $0,1,10,100,1000.88$

5.16 Isocontorno do Parâmentro de Hubble em função da massa dos neutrinos e o desvio para o vermelho.

5.17 Gráfico da diferença fracionária do Parâmentro de Hubble em função da massa dos neutrinos e do desvio para o vermelho em três dimensões.

5.18 Gráfico da diferença fracionária da razão do Parâmetro de Hubble em função da massa dos neutrinos. 


\section{Lista de tabelas}

2.1 Valores das massas dos neutrinos.

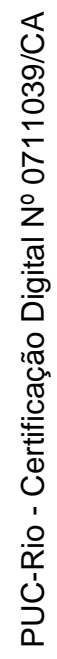




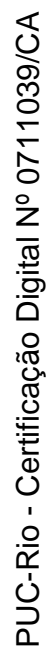

"Para Azucena y Jasmin, dos hermosas flores que alegran y le dan sentido a mi vida."

Alex... 